\title{
Implementation Experiences with Improving Safe Medication Practices for Oxytocin and Magnesium Sulfate During Labor and Delivery
}

\author{
Kayla B. Gray, BA ${ }^{1}$; Asta V. Sorensen, MA ${ }^{1 *}$; Samantha A. Sommerness, DNP, APRN, CNM²; \\ Kristi K. Miller, MS, RN³; Hannah Margaret Clare, BAㅜ; Kamila Mistry, PhD4; Leila Kahwati, MD¹
}

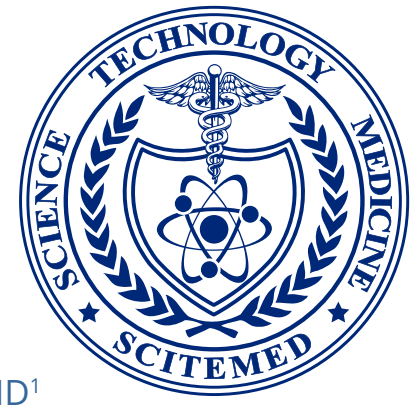

\author{
RTI International, Research Triangle Park, North Carolina, USA \\ 2 University of Minnesota School of Nursing, Minneapolis, Minnesota, USA \\ ${ }^{3}$ Retired Partner of Medical Teamwork Consultants LLC, Edina, Minnesota, USA \\ ${ }^{4}$ Agency for Healthcare Research and Quality, Rockville, Maryland, USA
}

\begin{abstract}
Objectives: Oxytocin and magnesium sulfate are high-alert medications with risks for significant patient harm. This paper describes experiences of 25 Labor and Delivery (L\&D) units which sought to apply safety science principles such as standardization, learning from defects, independent checks, teamwork and communication, and in situ simulation training for safe administration of oxytocin and magnesium sulfate. This effort was part of the Agency for Healthcare Research and Quality's Safety Program for Perinatal Care.

Methods: Use of safety science principles was monitored using a web-based reporting tool prior to program implementation, during, and 10 months after the start of implementation. Implementation experiences were assessed using semi-structured interviews and analyzed using qualitative thematic analysis techniques.

Results: L\&D units successfully applied safety science principles to medication administration. They achieved 100\% standardization and made substantial progress in creating independent checks ( $82 \%$ for oxytocin and $78 \%$ for magnesium sulfate) and improving teamwork and communication ( $95 \%$ for oxytocin and $78 \%$ for magnesium sulfate). Learning from defects was maximized for oxytocin (91\%) and less so for magnesium sulfate (67\%). The least commonly applied principle was the use of in situ simulations, with only a third of participants utilizing such trainings for safe medication practices.

Conclusions: Concurrent application of safety science principles to medication administration may offer a novel and effective approach to enhancing medication safety on L\&D units.
\end{abstract}

\section{Introduction}

The National Academy of Medicine reports that medication-related patient injuries are the leading type of adverse event in the in-patient setting and estimates that on average, hospitalized patients experience at least one medication error per day [1]. The Institute for Safe Medication Practices lists both intravenous (IV) oxytocin and magnesium sulfate infusions as two of twelve high-alert medications bearing a heightened risk of causing significant patient harm when used in error [2]. Because of the frequent use of these medications on labor and delivery (L\&D) units, a robust, unit-wide approach to preventing medication errors is needed to reduce adverse maternal and neonatal outcomes related to the use of these medications [3-5].

Oxytocin is most commonly used to induce or augment labor and to prevent postpartum hemorrhage. In 2015 , approximately $23.8 \%$ of all births in the United States involved induction of labor [6], and oxytocin is among the most commonly used drugs for labor induction [7]. Errors associated with oxytocin are most frequently dose-related, including wrong dose or rate, or involve oxytocin mixtures being mistaken for saline fluids during IV blousing [4]. In one state, oxytocin was the most commonly implicated medication in wrong-dose/overdose medication errors on L\&D units over the years 2004 to 2009 [8].

Magnesium sulfate is indicated for the prevention and control of seizures in preeclampsia and eclampsia, for neuroprotection of the fetus before anticipated early preterm delivery, and for the postponement of premature delivery between 24 and 34 weeks of gestational age [9]. Immediate consequences of magnesium sulfate toxicity may include nausea, vomiting, flushing, urinary retention, hypotension, bradycardia, loss of variability in the fetal heart rate, depressed neuromuscular function, and central nervous system depression leading to respiratory arrest and death $[3,10]$. Prolonged use of magnesium beyond 48 hours may result in untoward effects on neonatal bone mineralization, and use outside of indications subjects patients to all of the risks of use, without any clear benefit. Errors related to magnesium use often include errors in solution mixing and dosing, particularly related to using the same IV bag for both the bolus and infusion doses $[3,11,12]$. Such errors are further compounded by poor monitoring with failure to recognize early signs of magnesium toxicity, and an uncoordinated and delayed rescue response.

Ensuring the safe use of oxytocin and magnesium sulfate requires systematic approaches that may include policies and efforts to standardize processes, staff training and education, and environment and workflow redesign, all of which contribute to high-reliability care teams and a culture of patient safety [13-15]. Typical strategies for reducing medication errors include separate storage and clear labeling of all IV fluids and medications, the use of premixed IV medication solutions, the use of calibrated infusion pumps, and IV administration sets that label all tubing and clearly distinguish bolus from maintenance infusions $[3,8,11]$. In addition, limits on the use of verbal orders, frequent double checks of IV set ups at all shift changes or patient transitions, and appropriate nursing staff ratios to ensure close patient monitoring are recommended to 
prevent errors from occurring and mitigate the consequences of errors that do occur $[8,11]$. Although these best practices for safe use of medications can be operationalized into protocols and checklists $[16,17]$, implementation of such tools can be challenging in the absence of a unit culture that places a high priority on patient safety.

This article describes L\&D unit experiences with implementation of strategies for safe oxytocin and magnesium sulfate administration as part of the Agency for Healthcare Research and Quality's (AHRQ) Safety Program for Perinatal Care (SPPC) [18]. Although data are not available to indicate that these medications cause more errors than other high-alert medications used in perinatal care, these two medications were selected because the consequences of errors from use of the medications are the most serious for patients. Other high alert medications used in L\&D follow widely adopted evidence-based protocols, which are not available for oxytocin [19].

\section{Methods for Program Design and Implementation}

The AHRQ SPPC was designed to improve patient safety, team communication, and quality of care on L\&D units. The program is organized around three pillars of interventions: improving unit safety culture through teamwork and communication; using perinatal-specific safety strategies for common obstetric processes and in response to obstetric emergencies; and mutual reinforcement of the first two pillars with in situ simulation training. Safe medication administration is one of seven available strategies. The SPPC builds on several related AHRQ patient safety initiatives, including Team Strategies and Tools to Enhance Performance and Patient Safety (TeamSTEPPS ${ }^{\circledR}$ ) teamwork and communication program, Comprehensive Unit-based Safety Program (CUSP), and Patient Safety and Medical Liability Initiatives [18,20-23]

Key CUSP safety science principles include standardization, use of independent checks, and learning from defects [22]; these principles were used to inform program tools and resources related to developing unit policies and procedures for the safe administration of oxytocin and magnesium sulfate. Standardization strategies included establishment of standard criteria for use; uniform and premixed preparations and labeling; standard dosing protocols; calibrated infusion pumps; uniform parameters for monitoring and provider notification; and avoidance of high-risk practices, such as using the magnesium sulfate maintenance infusion for a manual bolus. Independent checks included assessing appropriateness of use by staff other than the ordering provider, double checks of IV setup and dose during patient or staff transitions, and limits on verbal orders and use of preprinted or programmed order sets. Learning from defects included team evaluation of processes and informal and formal debriefings of clinical or operational events or situations that should not have happened to achieve transparency and organizational learning [24]. SPPC implementing units also worked to improve teamwork and communication related to safe administration of oxytocin and magnesium sulfate, and conducted in situ simulations focused on early recognition and intervention to mitigate the clinical consequences of errors related to these medications.

Participating L\&D units attended five training webinars followed by 10 months of implementation, a duration determined by practical constraints. During this time, unit staff attended monthly technical assistance webinars and received virtual ad-hoc coaching and data feedback reports to support implementation.

\section{Evaluation Methods}

We used several data sources to evaluate SPPC implementation. We obtained hospital characteristics from the American Hospital Association Annual Member Survey and from data submitted by participating units [25]. As part of program monitoring, we used a web-based reporting form to collect data about the use of CUSP safety principles for oxytocin and magnesium sulfate administration prior to program implementation ("baseline"), during, and at 10 months after the start of implementation ("follow-up"). L\&D units reported the consistent use of CUSP safety principles using a 4-point Likert scale (not at all, slightly, somewhat, and mostly). To assess changes between baseline and follow-up, we calculated and compared the percentage of units reporting lower (i.e., responding "not at all" or "slightly") and higher (i.e., responding "somewhat" or "mostly") levels of consistent use. Quantitative data were analyzed using Stata version 14 (College Station, Texas, USA).

To assess implementation experiences, a team of six researchers trained and experienced in qualitative research conducted semi-structured interviews with L\&D unit teams by phone and in person. Interview questions used implementation research constructs based on the Consolidated Framework for Implementation Research (CFIR) [26] and sought to understand improvements units made in implementing key safety strategies for administration of oxytocin and magnesium sulfate. Interviews were audio-recorded and transcribed for further analysis and interpretation. We coded and analyzed interview transcripts using NVivo (Version 10) software using inductive and deductive qualitative analysis techniques [27], using CFIR constructs as the basis for initial list of codes [28]. The evaluation was not considered to be human subjects' research by the RTI International Institutional Review Board.

\section{Results}

Of the 46 L\&D units that participated in the program, 25 selected safe medication administrations as an improvement strategy: 16 focused on oxytocin, three focused on magnesium sulfate, and six focused on both. Hospital and L\&D unit characteristics as well as the patient populations of these sites are described in Table 1. The median number of L\&D unit beds was 10 (range 2 to 23) and the median number of hospital beds was 279 (range 42 to 875 ). Five were publicly owned hospitals and six had a graduate medical education program in obstetrics and gynecology. The median number of staff L\&D nurses providing care was 37 (range 13 to 95) and the median number of annual births was 1,367 (range 153 to $5,278)$. Ten units (40\%) offered Level 3 neonatal care.

Unit staff indicated they chose to focus on medication safety because they wanted to reduce variation in practices, streamline administration of these medications, and assure that they were meeting the

Table 1. Hospital and L\&D Unit Characteristics for Sites Implementing Perinatal Safety Strategies related to Safe Medication Administration*

\begin{tabular}{|l|l|}
\hline \multicolumn{1}{|c|}{ Hospital or L\&D Unit Characteristic } & \multicolumn{1}{|c|}{$\begin{array}{c}\text { Number or } \\
\text { Median }\end{array}$} \\
\hline Hospital Characteristics & \\
\hline Publicly owned, N (\%) & $5(20)$ \\
\hline Total hospital beds, median (range) & 279 (42 to 875) \\
\hline $\begin{array}{l}\text { Graduate medical education program for } \\
\text { Obstetrics/Gynecology, N (\%) }\end{array}$ & $6(24)$ \\
\hline L\&D Unit Characteristics & $10(2$ to 23) \\
\hline L\&D unit beds, median (range) & $1367(153$ to 5,278) \\
\hline Annual number of births, median (range) & $37(13$ to 95) \\
\hline L\&D staff nurses providing care, median (range) & $9(36)$ \\
\hline Level 1 basic neonatal care, N (\%) & $6(24)$ \\
\hline Level 2 specialty neonatal care, N (\%) & $10(40)$ \\
\hline Level 3 sub-specialty neonatal care, N (\%) & $26(25$ to 31) \\
\hline Mean age of L\&D unit patients, median (range) & $73 \%(30 \%$ to 100\%) \\
\hline $\begin{array}{l}\text { Mean percentage of L\&D patients that are } \\
\text { white, median (range) }\end{array}$ & $34 \%(5 \%$ to 89\%) \\
\hline $\begin{array}{l}\text { Mean percentage of L\&D patients covered by } \\
\text { commercial insurance, median (range) }\end{array}$ & $54 \%(10 \%$ to 90\%) \\
\hline $\begin{array}{l}\text { Mean percentage of L\&D patients covered by } \\
\text { medicaid, median (range) }\end{array}$ & \\
\hline
\end{tabular}

*The number of patients were 25 overall, though it may vary for selected characteristics. L\&D, labor and delivery; N, number. 


\section{ORIGINAL}

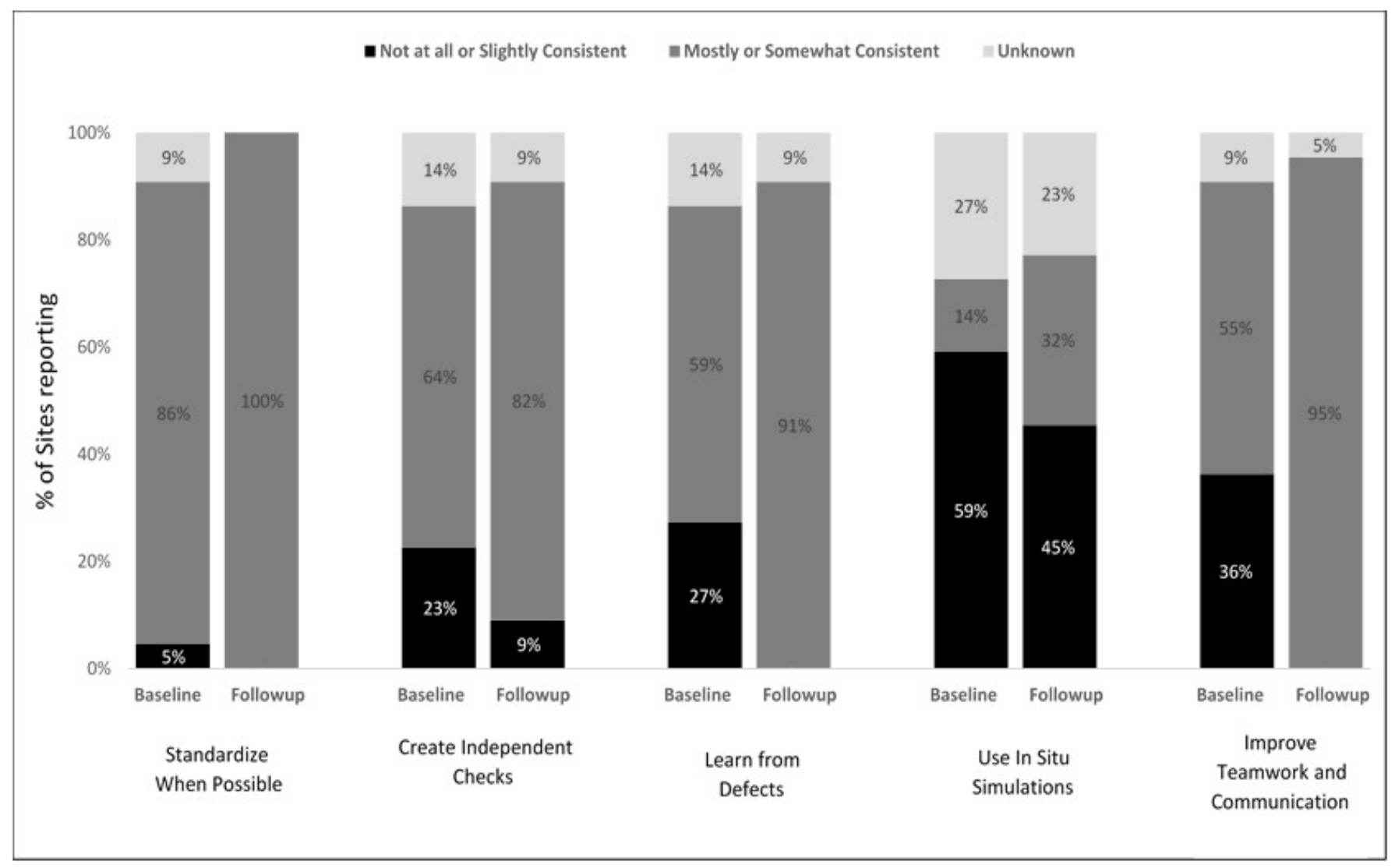

Figure 1. L\&D Units' level of reported consistency with CUSP safety science principles applied to safe medication administration of Oxytocin at baseline and follow-up ( $N=22)$. L\&D, labor and delivery; CUSP, comprehensive unit-based safety program.

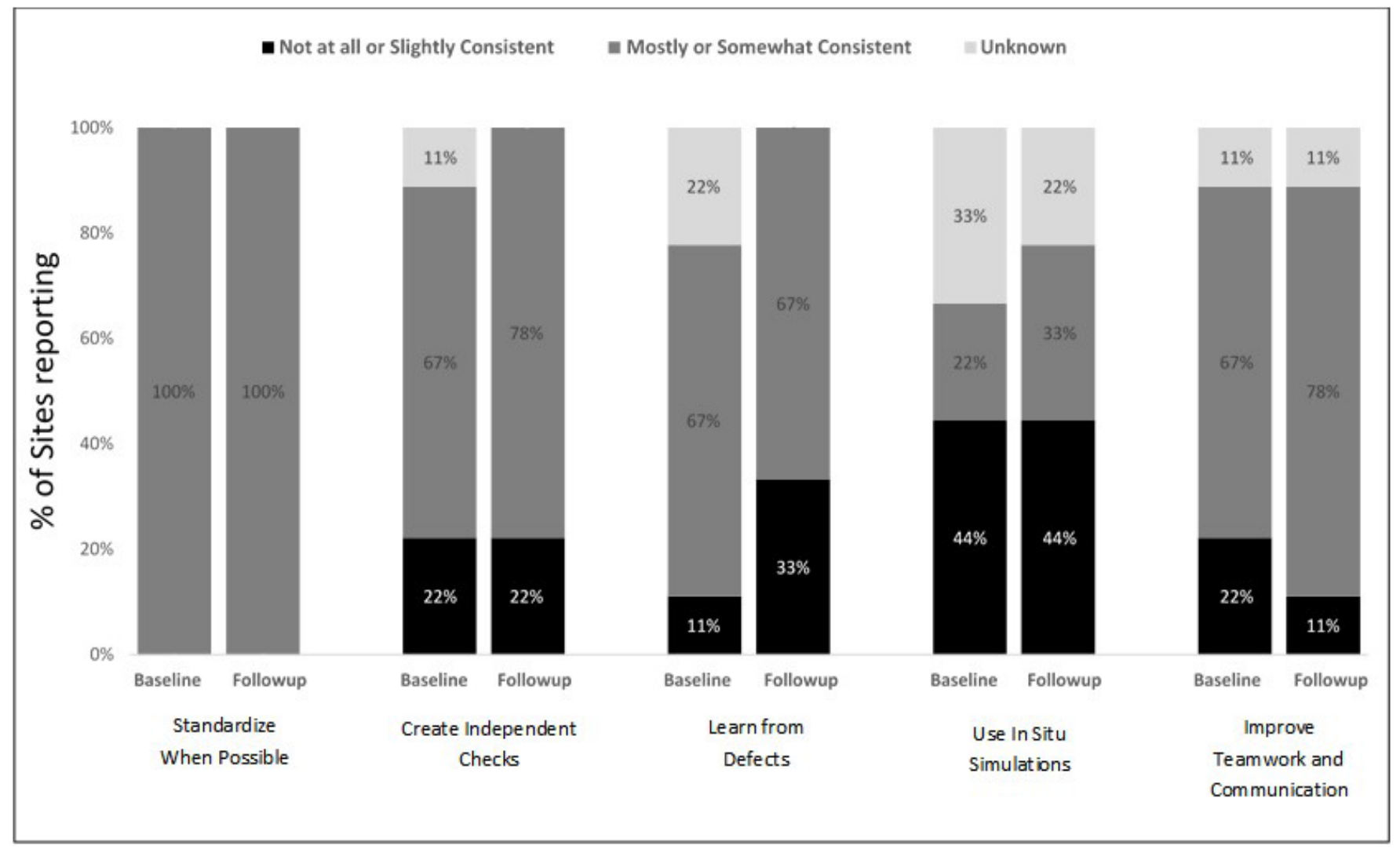

Figure 2. L\&D Units' level of reported consistency with CUSP safety science principles applied to safe medication administration of Magnesium Sulfate at baseline and follow-up ( $N=9)$. L\&D, labor and delivery; CUSP, comprehensive unit-based safety program. 
standards of care. Several others embarked on this effort in response to recent near misses and concerns about litigation. For example, staff of one unit shared that an obstetrics resident hung a bag of oxytocin and accidentally bolused it as post-partum hemorrhage prophylaxis before the baby was delivered. Staff from another unit described an incident where oxytocin was mistaken for an anesthetic drug when the oxytocin was set on top of the anesthesia cart. Such near misses served as drivers for change.

\section{Teamwork and Communication}

Units reported improvements in enhancing teamwork and communication for oxytocin administration, with consistent use increasing from 55\% at baseline to $95 \%$ at follow-up (Figure 1). Smaller increases in the consistent use of teamwork and communication were reported for magnesium sulfate, $67 \%$ at baseline to $78 \%$ at follow-up (Figure 2). Staff from several units reported that training in teamwork and communication began changing unit culture. For example, staff of one unit observed that using Team STEPPS techniques during discussions of oxytocin use removed the appearance that decisions were one person's opinion and guided decision making back to the unit's standardized oxytocin checklist. Improved teamwork and communication empowered nurses and improved their relationships with physicians and other unit staff. Several units shared that as result of the trainings, nurses were comfortable challenging authority and questioning changes in medication administration when in doubt:

\section{(...) What we did is we took that whole Team STEPPS approach and} we gave them that culture of safety to say "You know what, [when the questionable oxytocin strip] is in the yellow [category], this is what you're supposed to do. You're supposed to be at the bedside. You're supposed to turn off the [oxytocin]. I'm supposed to call ..." It gave them a chain of command. It gave them a guideline to set.

Culture change through improved teamwork and communication helped to overcome several implementation challenges, such as getting physician and leadership buy-in for policy changes, adapting existing electronic health record systems to document care according to new protocols, and managing new policy logistics. Nurse leaders observed changes in unit culture through the empowerment of nurses and their ability and willingness to challenge authority, increased situational awareness of staff during medication administration, and the effective engagement of physicians in formulating new policies. These changes resulted in improved team member engagement and an overall improvement in awareness about the importance of safe medication administration for patient safety.

\section{Standardization}

At baseline, $86 \%$ of all units reported somewhat or mostly consistent use of standardization with respect to their unit policies and procedures for the safe administration of oxytocin; this increased to $100 \%$ by the end of program implementation (Figure 1). For magnesium sulfate, all units reported somewhat or mostly consistent use of standardization at both baseline and follow-up (Figure 2).

Many units revised and standardized their medication policies and procedures. Some introduced a requirement for nurses to document fetal heart rate and the uterine contraction pattern every time oxytocin was adjusted. Others integrated an oxytocin policy into a policy on early elective inductions or developed a joint nursing and physician oxytocin policy. Some units changed order sets in electronic health records and placed a sheet summarizing the new procedure and instructions at each nursing station to serve as a checklist for staff actions.

Several units changed packaging or equipment to standardize delivery of oxytocin and magnesium sulfate. These changes included adding commercial software to the IV pumps or buying new "smart" pumps to control infusion rates and prevent errors. These changes also included incorporating tubing labels or color coding to distinguish different IV lines. The most common policy change for magnesium sulfate was instituting a new standard concentration in a smaller $500 \mathrm{~mL}$ bag for the bolus infusion, clearly delineating the bolus from the maintenance infusion.
For several units, the move to standardized dosing meant discussions with the pharmacy and obtaining approvals from relevant hospital committees. Such requests and approvals were typically received with some resistance. For oxytocin, units experienced resistance from pharmacy to change to a standard bagged solution. To change magnesium sulfate packaging, units experienced difficulties in getting pharmacy cooperation to change to $500 \mathrm{~mL}$ bags or change the order sets.

A few L\&D units encountered difficulties getting administrative support or buy-in for policy changes related to oxytocin administration. One unit was forced to rescind a new, low-dose oxytocin policy after one of its private obstetric groups opposed the change and formally complained. Further discussions with the group resulted in a more specific policy that was readily accepted. Another unit encountered pushback from their healthcare system when an attempt to standardize the approach to ordering oxytocin required a system-level change. This unit commented that although it had two formal, standard ways of ordering oxytocin, a third way continued to exist, which was described as "they [the physician] can do it the way they want."

Many units standardized medication dosing. For oxytocin, this meant creating a shared understanding of dosing orders between nurses and physicians, so that "physicians don't have the opportunity to order something that the nurses can't execute." Such processes also led to more standardized approach to the use of other medications:

We've not only put out the policies and procedures, we carried the structure so that the right thing to do is the easy thing to do. (...) You create cross checks by having individual members of the team with different disciplines doing the same thing.

One L\&D unit noted that implementing changes to the administration of these medications was a massive logistical undertaking-but ultimately a huge win-that included developing a single standardized bag for oxytocin, figuring how to move the product through the system, and getting it to fit in the medication dispensing system drawer.

\section{Using Independent Checks}

Consistent use of independent checks for the safe administration of oxytocin increased from $64 \%$ at baseline to $82 \%$ at follow-up (Figure 1). Similar improvements were achieved for magnesium sulfate $(67 \%$ at baseline to $78 \%$ at follow-up) (Figure 2 ).

Unit staff built in independent checks for medication administration by using checklists and deployment of double-checks at initiation, dosage change, and shift change for magnesium sulfate. One unit developed a protocol for magnesium sulfate to track duration from the time the order for medication was placed until administration. Such tracking revealed that sometimes delays in starting the medication are warranted (e.g., when a nurse takes time to provide patient education); however, other causes of delay, such as limited staffing or lack of IV access, warranted attention.

Cross-checking oxytocin infusion through independent checks by L\&D and postpartum staff ensured that postpartum patients received their oxytocin infusion using the same procedures as those for L\&D. Units implemented independent checks by using checklists and algorithms for medication administration for several procedures, such as during Category II fetal heart tracings. Checklists were maintained and a standard algorithm was put in place for Category II fetal tracings. One unit that required documentation every time the oxytocin dose was increased reported more L\&D staff awareness and fewer dosage increases.

One unit implemented a clinical decision support tool for managing patients receiving oxytocin for labor induction or augmentation. Leaders of this unit observed that nurses had no trouble with patient care in the green zone of the decision support tool ("everything is good") and red zone ("stop oxytocin"); however, they experienced a lot of delays in patient treatment and interventions in the yellow zone ("manage oxytocin with caution"). Unit staff used this tool as a communication tool for questioning, and sometimes challenging, physician decisions, escalating the chain of command, and standardizing oxytocin management among private physicians practicing on the unit: 
(...) when I start getting concerned about how the Pitocin is being titrated at that time, I go back to the checklist to say, per the checklist, we need to be careful that we're not increasing it. Let's take a look. It's a conversation piece (...) which brings it back to the TeamSTEPPS so I can take that out and say this isn't my opinion. This isn't that I'm trying to be harsh about this. This is our standard. (...) This is what we're all held to.

\section{Other Safety Science Principles}

Units enhanced the consistent use of learning from defects related to safe oxytocin administration from $59 \%$ at baseline to $91 \%$ at follow-up, whereas units reported no change in the consistent use of this safety science principle with respect to magnesium sulfate administration (67\% at baseline and follow-up) (Figures 1 and 2). Several units conducted chart audits and case reviews to identify gaps in their safe medication administration policies and protocols. Ongoing chart audits conducted by some units allowed their leadership to monitor and sustain adherence to changes. Staff of one unit shared that consistent debriefings related to oxytocin management improved relationships between nurses and physicians.

\section{In Situ Simulation}

At baseline, only 3 units (14\%) reported conducting in situ simulations related to oxytocin and $22 \%(N=2)$ reported conducting simulations related to magnesium sulfate; consistent use of simulations increased from baseline to follow-up for both medications (Figures 1 and 2). For example, one L\&D unit that implemented in situ simulations for oxytocin administration focused on when to start and stop oxytocin, when to restart oxytocin and at what dose, and the use of an oxytocin checklist. Unit simulations for magnesium sulfate included responding to magnesium toxicity and preeclampsia/eclampsia. In situ simulations for medication safety were an effective way to re-enforce the checklist use and to assure that unit staff were consistently following the new policies:

(...) it was very interesting how many staff members thought they knew the [oxytocin] checklist. We would often wait to see if they went to the resource or if they were simply trying to recall it, and if they were recalling it correctly or their interpretation of it. (...) That was a huge insight that we got from group to group, to watch how nurses interacted with the tools that were available.

\section{Discussion}

Most L\& D units successfully applied safety science principles to oxytocin and magnesium sulfate administration. For oxytocin, improvements were noted for all five safety science principles. For magnesium sulfate administration, improvements were observed in three of the five safety elements; the consistent use of standardization was already reported by $100 \%$ of participating units at baseline and no changes were reported in the consistent use of learning from defects.

L\&D units improved medication administration processes through implementation of checklists, algorithms, flow sheets, tracking forms, standard order sets, set dosages, standard infusion bag size for bolus or maintenance infusion, and smart pumps. Tools and strategies enhancing standardization and independent checks are known to improve maternal outcomes $[16,19]$. As it has been well documented in the literature $[29,30]$, no single approach or tool is generally responsible for sustained improvement. This evaluation suggests that use of safety science principles from the CUSP framework can enhance implementation of specific tools, protocols, and procedures related to medication administration. Standardization of oxytocin and magnesium sulfate management reduced variation in the use of these medications among physicians and gave nurses communication tools to employ when concerned about a physician's medication administration decisions.

Despite a high level of reported use of these principles at baseline, interview findings suggest that units did, in fact, make further substantial improvements to their processes and procedures to advance safety science principles. We hypothesize that units may not have fully realized the potential for full applications of these principles at the time of baseline reporting, resulting in artificially high reported levels of use at the baseline. For instance, a nurse manager may have perceived existing order sets and unit processes as "standardized"; however, once a unit began program implementation, they realized additional ways to enhance the application of standardization and independent checks.

The most room for improvement remains in the use of in situ simulations, which had very low consistent use at baseline with limited uptake at follow-up. This could be due to several factors, such as desire to focus on strategies that were easier to implement, limited perceived applicability and visibility of value for such trainings in the context of medication administration, limited financial or staff resources to plan and implement this type of training, or need for more time to initiate such effort. Lastly, developing a program of in situ simulation typically takes several months, thus units may have continued planning for and running in situ simulations using the scripted simulations provided through the program following the formal end date of the implementation period.

This study has several limitations. First, we relied on L\&D units to self-report consistency with the use of safety science principles as part of their SPPC implementation at baseline and follow-up. Second, the period of observation following the start of implementation was only 10 months. Based on our experience with L\&D units, the first several months comprised "startup" activities, with little change to existing processes or policies. Third, the small number of units that chose to implement these strategies limits the generalizability of results. The overall study outcomes related to adverse maternal and neonatal events are reported in the AHRQ Safety Program for Perinatal Care Summary Report [31].

\section{Conclusion}

Concurrent application of safety science principles to medication administration may offer a novel and effective approach to enhancing medication safety on L\&D units. Several other high-risk medications (e.g., epinephrine, insulin, nitroprusside, potassium chloride, promethazine) and medication delivery methods (venous access, IV and epidural tubing, infusion pumps) used during labor and delivery can benefit from application of similar strategies and approaches [32,33]. Future research might benefit from a study with a larger number of L\&D units and that entails a longer implementation and sustainment period along with a comparison group to enable improvements to be attributed to implementation of the safety program.

\author{
Article Information \\ * Correspondence: Asta V. Sorensen, MA. RTI International, 3040 E. Cornwal- \\ lis Road, P.O. Box 12194, Research Triangle Park, NC 27709, USA. \\ Email: asorensen@rti.org
}

Received: Apr. 11, 2018; Accepted: Jul. 05, 2018; Published: Aug. 13, 2018

DOI: 10.24983/scitemed.rwh.2018.00075

Copyright $\odot 2018$ The Author (s). This is an open-access article distributed under the terms of the Creative Commons Attribution 4.0 International License (CC-BY).

Funding: This project was funded by the Agency for Healthcare Research and Quality under contract No. HHSA2902010000241, Task Order 3 to RTI International.

\section{Conflicts of Interest: None}

Additional Note: Appendix A, In-situ simulation tools for magnesium sulfate toxicity are included as supplemental digital content.

\section{Acknowledgments}

The authors would like to recognize the contributions of Brenda Harding for her guidance to this project, Stephanie Teixeira-Poit, Elizabeth Pleasants, and Sara Jacobs for analytic support with the process survey, Jill 
McArdle, Katrina Burson, and Beth Lasater for coordination of unit activities, McKenzie Armstrong for assistance with literature review, Program faculty for their input and review of program content and trainings, and Program participants for their time and dedication to safety and quality improvements on their units.

\section{Keywords}

Labor and delivery; magnesium sulfate; maternal health; medication safety; neonatal health; oxytocin; patient safety; perinatal health; quality improvement.

\section{References}

1. Institute of Medicine. Preventing medication errors. Washington, DC: National Academies Press, 2007.

2. Institute for Safe Medication Practices. List of high-alert medications. 2014. Available at: https://www.ismp.org/tools/highalertmedications.pdf.

3. Simpson KR, Knox GE. Obstetrical accidents involving intravenous magnesium sulfate: recommendations to promote patient safety. MCN Am J Matern Child Nurs 2004;29(3):161-169.

4. Simpson KR, Knox GE. Oxytocin as a high-alert medication: implications for perinatal patient safety. MCN Am J Matern Child Nurs 2009;34(1):8-15.

5. Krening CF, Rehling-Anthony K, Garko C. Oxytocin administration: the transition to a safer model of care. J Perinat Neonatal Nurs 2012;26(1):15-24.

6. Martin JA, Hamilton BE, Osterman MJ, et al. Births: final data for 2015. Natl Vital Stat Rep 2017;66(1):1.

7. American College of Obstetricians and Gynecologists. Practice bulletin No. 107: induction of labor. Obstet Gynecol 2009;114(2 Pt 1):386397.

8. Pennsylvania Patient Safety Advisory. Medication errors in labor and delivery: reducing maternal and fetal harm. 2009. Available at: http:// patientsafetyauthority.org/ADVISORIES/AdvisoryLibrary/2009/ dec16_6(suppl1)/Pages/01.aspx.

9. American Congress of Obstetricians and Gynecologists Committee of Obstetric Practice. Committee opinion number 652. Magnesium sulfate use in obstetrics. January 2016, 2016. Available at: http://www. acog.org/Resources-And-Publications/Committee-Opinions/Committee-on-Obstetric-Practice/Magnesium-Sulfate-Use-in-Obstetrics.

10. Lu JF, Nightingale $\mathrm{CH}$. Magnesium sulfate in eclampsia and pre-eclampsia: pharmacokinetic principles. Clin Pharmacokinet 2000;38(4):305-314.

11. Simpson KR. Minimizing risk of magnesium sulfate overdose in obstetrics. MCN Am J Matern Child Nurs 2006;31(5):340.

12. Grissinger $M$. Preventing magnesium toxicity in obstetrics. $P \& T$ 2009;34(8):403.

13. Riley W, Davis SE, Miller KK, McCullough M. A model for developing high-reliability teams. J Nurs Manag 2010;18(5):556-563.

14. Cherouny PH, Federico FA, Haraden C, et al. Idealized design of perinatal care. IHI innovation series white paper. Cambridge, MA: Institute for Healthcare Improvement, 2005. Article Link: https://www. mnhospitals.org/Portals/0/Documents/ptsafety/perinatal/whitepaper-perinatal.pdf

15. Simpson KR, Knox GE, Martin M, et al. Michigan Health \& Hospital Association Keystone Obstetrics: a statewide collaborative for perinatal patient safety in Michigan. Jt Comm J Qual Patient Saf 2011;37(12):544-552.

16. Arora KS, Shields LE, Grobman WA, et al. Triggers, bundles, protocols, and checklists--what every maternal care provider needs to know. Am J Obstet Gynecol 2016;214(4):444-451.
17. Agency for Healthcare Research and Quality. Standardized protocols and processes enhance compliance with recommended care, improve staff perceptions of patient safety at a birthing center. 2009. Available at: http://www.innovations.ahrq.gov/content.aspx?id=2362.

18. Agency for Healthcare Research and Quality. AHRQ safety program for perinatal care. May 2015, 2016. Available at: http://www.ahrq. gov/professionals/quality-patient-safety/hais/tools/perinatal-care/ index.html.

19. Clark S, Belfort M, Saade G, et al. Implementation of a conservative checklist-based protocol for oxytocin administration: maternal and newborn outcomes. Am J Obstet Gynecol 2007;197(5):480e1-5.

20. Agency for Healthcare Research and Quality. TeamSTEPPS: strategies and tools to enhance performance and patient safety. May 2016, 2016. Available at: http://www.ahrq.gov/professionals/education/curriculum-tools/teamstepps/index.html.

21. Agency for Healthcare Research and Quality. Comprehensive unitbased safety program. June 2015, 2016. Available at: http://www. ahrq.gov/professionals/education/curriculum-tools/cusptoolkit/index.html.

22. Agency for Healthcare Research and Quality. Patient safety \& medical liability reform initiative. October 2014, 2016. Available at: http:// www.ahrq.gov/professionals/quality-patient-safety/patient-safety-resources/resources/liability/index.html.

23. Agency for Healthcare Research and Quality. Demonstration grants final evaluation report. May 2016, 2016. Available at: http://www. ahrq.gov/professionals/quality-patient-safety/patient-safety-resources/resources/candor/demo-program-eval/demo-grants.html.

24. Agency for Healthcare Research and Quality. CUSP toolkit: learn from defects tool. 2012. Available at: http://www.ahrq.gov/professionals/ education/curriculum-tools/cusptoolkit/toolkit/learndefects.html.

25. American Hospital Association. AHA Annual Survey Database. 2012. Available at: https://www.ahadataviewer.com/additional-data-products/AHA-Survey/.

26. Damschroder LJ, Aron DC, Keith RE, et al. Fostering implementation of health services research findings into practice: a consolidated framework for advancing implementation science. Implement Sci 2009;7(4):50.

27. Patton MQ. Qualitative research and evaluation methods. Thousand Oaks, CA: Sage Publications, 2002.

28. Boyatzis RE. Transforming qualitative information: thematic analysis and code development. Thousand Oaks, CA: SAGE Publishers, 1998.

29. Leape LL. The checklist conundrum. N Eng/J Med 2014;370(11):10631064.

30. Urbach DR, Govindarajan A, Saskin R, et al. Introduction of surgical safety checklists in Ontario, Canada. N Engl J Med 2014;370(11):10291038.

31. Sorensen AV, Webb J, Clare HM, et al. AHRQ Safety Program for Perinatal Care: Summary Report. Rockville, MD: Agency for Healthcare Research and Quality; 2017. Available from: www.ahrq.gov/perinatalsafety.

32. Institute for Safe Medication Practice. Your high-alert medication listrelatively useless without associated risk-reduction strategies. 2013. Available at: https://www.ismp.org/resources/your-high-alert-medication-list-relatively-useless-without-associated-risk-reduction

33. Kacmar RM, Mhyre JM. Obstetric anesthesia patient safety: Practices to ensure adequate venous access and safe drug administration during transfer to the operating room for emergency cesarean delivery. June 29, 2018, 2015. Available at: https://www.apsf.org/article/obstetric-anesthesia-patient-safety-practices-to-ensure-adequate-venous-access-and-safe-drug-administration-during-transfer-to-the-operating-room-for-emergency-cesarean-delivery/. 